\title{
Proteomic profiling of the endogenous peptides of MRSA and MSSA
}

\author{
Haixia Tu ${ }^{\text {Equal frrst author, } 1}$, Fei Xu ${ }^{\text {Equal first author, } 2}$, Yiwei Cheng ${ }^{3}$, Qianglong Pan ${ }^{1}$, Xiao Cai ${ }^{1}$, Shouxing Wang ${ }^{1}$, Shuting Ge $^{3}$, \\ Min Cao ${ }^{1}$, Dongming Su ${ }^{\text {Corresp., } 1}$, Yan Li ${ }^{\text {Corresp. } 1}$ \\ ${ }^{1}$ Center of Pathology and Clinical Laboratory, Sir Run Run Hospital, Nanjing Medical University, Nanjing, Jiangsu Province, China \\ 2 Blood Transfusion Department, Nanjing First Hospital, Nanjing Medical University, Nanjing, Jiangsu Province, China \\ 3 School of Basic Medicine, Nanjing Medical University, Nanjing, Jiangsu Province, China \\ Corresponding Authors: Dongming Su, Yan Li \\ Email address: sudongming@njmu.edu.cn, yanli@njmu.edu.cn
}

Staphylococcus aureus is a Gram-positive bacterium that can cause diverse skin and soft tissue infections. Methicillin-resistant Staphylococcus aureus (MRSA) can cause more severe infections than methicillin-susceptible Staphylococcus aureus (MSSA).

Nevertheless, the physiological and metabolic regulation of MSSA and MRSA has not been well studied. In light of the increased interest in endogenous peptides and recognition of the important roles that they play, we studied the endogenous peptidome of MSSA and MRSA. We identified 1065 endogenous peptides, among which 435 were differentially expressed (DE) , with 292 MSSA-abundant endogenous peptides and 35 MRSA-abundant endogenous peptides. MSSA-abundant endogenous peptides have significantly enriched "VXXXK" motif of at the C-terminus. MSSA-abundant endogenous peptides are involved in penicillin-binding and immune responses, whereas MRSA-abundant endogenous peptides are associated with antibiotic resistance and increased toxicity. Our characterization of the peptidome of MSSA and MRSA provides a rich resource for future studies to explore the functional regulation of drug resistance in $S$. aureus and may also help elucidate the mechanisms of its pathogenicity and the development of treatments. 
1 Proteomic profiling of the endogenous peptides of MRSA and MSSA

2 Haixia $\mathrm{Tu}^{1}$, Fei Xu${ }^{2}$, Yiwei Cheng ${ }^{3}$, Qianglong $\mathrm{Pan}^{1}$, Xiao Cai ${ }^{1}$, Shouxing Wang ${ }^{1}$, Shuting $\mathrm{Ge}^{3}$,

3 Min $\mathrm{Cao}^{1}$, Dongming $\mathrm{Su}^{1, *}$, Yan $\mathrm{Li}^{1, *}$

$4 \quad{ }^{1}$ Center of Pathology and Clinical Laboratory, Sir Run Run Hospital, Nanjing Medical

5 University, Nanjing, China

$6 \quad{ }^{2}$ Blood Transfusion Department, Nanjing First Hospital, Nanjing Medical University,

$7 \quad$ Nanjing,China

$8{ }^{3}$ School of Basic Medicine, Nanjing Medical University, Nanjing, China

9 *Corresponding Author:

$10 \quad$ Yan $\mathrm{Li}^{1}$

11109 Longmian Avenue, Jiangning District, Nanjing, Jiangsu Province, 211100, China

12 Email address:yanli@njmu.edu.cn (Y.L.)

13 DongmingSu ${ }^{1}$

14109 Longmian Avenue, Jiangning District, Nanjing, Jiangsu Province, 211100, China

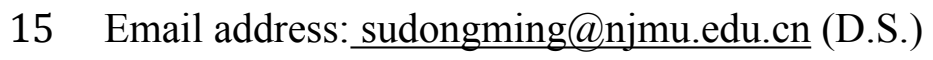


16 Haixia Tu and Fei Xu have contributed equally to this work and share first authorship.

17 Key words: Staphylococcus aureus; differential endogenous peptidome; protein; mass

18 spectrometry; MRSA

19 Abstract Staphylococcus aureus is a Gram-positive bacterium that can cause diverse skin and

20 soft tissue infections. Methicillin-resistant Staphylococcus aureus (MRSA) can cause more

21 severe infections than methicillin-susceptible Staphylococcus aureus (MSSA). Nevertheless, the

22 physiological and metabolic regulation of MSSA and MRSA has not been well studied. In light

23 of the increased interest in endogenous peptides and recognition of the important roles that they

24 play, we studied the endogenous peptidome of MSSA and MRSA. We identified 1065

25 endogenous peptides, among which 435 were differentially expressed (DE), with 292 MSSA-

26 abundant endogenous peptides and 35 MRSA-abundant endogenous peptides. MSSA-abundant

27 endogenous peptides have significantly enriched "VXXXK" motif of at the C-terminus. MSSA-

28 abundant endogenous peptides are involved in penicillin-binding and immune responses,

29 whereas MRSA-abundant endogenous peptides are associated with antibiotic resistance and

30 increased toxicity. Our characterization of the peptidome of MSSA and MRSA provides a rich

31 resource for future studies to explore the functional regulation of drug resistance in S. aureus and

32 may also help elucidate the mechanisms of its pathogenicity and the development of treatments.

Abbreviations: DE, differentially expressed; MSSA-AEP ,MSSA-abundant endogenous peptides; MRSA-AEP, MRSA-abundant endogenous peptides. 
34 Staphylococcus aureus (S. aureus), a Gram-positive bacterium, is a common cause of infections

35 in human worldwide and a significant threat to modern health care systems. As an opportunistic

36 nosocomial and community-associated pathogen, it is a normal part of human flora, covering

$3730 \%$ of the population, and is able to cause diverse skin and soft tissue infections, including

38 pimples, boils, cellulitis, folliculitis, impetigo, scalded skin syndrome, and abscesses, but also

39 cause severe diseases, including bacteremia, acute endocarditis, and meningitis (Monteiro et al.,

40 2012).

41 The overuse of antibiotics can lead to the development of drug-resistant $S$. aureus, such as

42 methicillin-resistant Staphylococcus aureus (MRSA), which can evade immune responses and

43 reproduce in human hosts (Singh et al., 2020). Aside from being distinguished from methicillin-

44 susceptible Staphylococcus aureus (MSSA) in their resistance to antibiotics, MRSA also differ in

45 their phenotype and metabolic pathways (Garcia et al., 2017). Previous studies have shown that

46 MRSA have significantly higher biofilm formation abilities than MSSA, as they can easily form

47 small colonies (Akil and Muhlebach, 2018) that colonize for long periods (Jeong et al., 2020)

48 and can develop thickened cell walls (Garcia et al., 2017). MRSA infection can lead to more

49 serious clinical symptoms, inflammatory reactions, and multi-drug resistance (Zhang et al.,

50 2019). It is more common in patients with nosocomial infections, catheter-related infections, or 
51 renal insufficiency, all of which require increased medical resources to treat infections. Thus,

52 MRSA infection is a major global health problem (Monteiro et al., 2012).

53 Given the importance of MRSA and MSSA as human pathogens, numerous proteomics studies

54 have been conducted to characterize differences in the composition of their proteins (Otto et al.,

55 2014). For example, Cordwell et. al. used 2-DE gel-based proteomics methods and identified 377

56 proteins of $S$. aureus. The proteins in MRSA included alkali shock protein 23 (Asp23) and the

57 cold-shock protein CspABC, which are not present in MSSA (Cordwell et al., 2002). Ji X et. al.

58 used an iTRAQ-based LC-MS/MS quantitative proteomics approach to study the effects of

59 emodin on MRSA and MSSA at the molecular level (Ji et al., 2017). However, nearly all

60 previous studies have focused on large proteins produced in S. aureus.

61 With the development of high-throughput technologies, an increasing number of studies have

62 focused on the peptidome, which includes endogenous peptides and can be characterized by

63 peptidomics in a high-throughput way (Romanova and Sweedler, 2015). Although endogenous

64 peptides are small in length, they can also have important functions (Carter, 2012; Cui et al.,

65 2016; Wolf et al., 2011). In eukaryotes, the peptidome has been shown to change during the

66 epithelial-mesenchymal transition of renal epithelial cells (Kanlaya and Thongboonkerd, 2018).

67 The neuropeptides can mediate cell-cell signaling and are potential therapeutic targets (Fricker,

68 2018). In S.aureus, pheromone peptides can cause activation of virulence regulatory factor agr,

69 leading to the early reproduction and infection spread of S.aureus (Otto, 2001). S.aureus can 
70 produce formylated peptides, which play important roles in S.aureus arthritis and may be used

71 for preventing joint destruction in S.aureus arthritis (Gjertsson et al., 2012). Phenolic soluble

72 modules (PSMs) are a family of amphiphilic short peptides produced by S.aureus. PMS can

73 cause the dissolution of human cells, stimulate the inflammatory response, and promote the

74 formation of biofilm in osteomyelitis (Cheung et al., 2014). Thus, systematic analysis of the

75 endogenous peptidome of $S$. aureus could provide useful insights into its physiology and

76 regulatory networks.

77 Here, we performed high-throughput liquid chromatography-mass spectrometry (LC-MS)

78 analysis of MSSA and MRSA and identified 1065 endogenous peptides from 282 precursor

79 proteins. After filtering mass spectrum data,, and 435 differentially expressed (DE) peptides

80 were obtained. 327 DE peptides of MSSA and MRSA were extracted for further study. The

81 abundance and diversity of DE endogenous peptides in S. aureus indicate that the regulation of

82 its physiological metabolism is complex. DE endogenous peptides of MSSA and MRSA may be

83 closely related to drug resistance and pathogenicity. We analyzed the endogenous peptides of

84 MRSA and MSSA by proteomics, aiming to provide a guidance for further mechanistic studies

85 and treatments of $S$. aureus. We believe that further research on endogenous peptides will

86 enhance our understanding of their roles in pathogenicity as well as their interaction with the

87 human body.

88 Materials and Methods 


\section{Sample preparation}

90 In this study, MSSA (ATCC 29213) and MRSA (ATCC 43300) samples were purchased from

91 the American Type Culture Collection. Three S. aureus clinical isolates were from the

92 Microbiology Department of Sir Run Run Hospital, Nanjing Medical University, and were

93 identified as MRSA by the BD Phoenix 100 Automated Microbiology System. Three clones

94 from MSSA (ATCC 29213 strains), three clones from MRSA (ATCC 43300 strains), and three

95 above-mentioned $S$. aureus clinical MRSA isolates were prepared for proteomic analysis. They

96 were placed onto blood agar plates and grown at $37^{\circ} \mathrm{C}$. The colonies were then cultured in 250

$97 \mathrm{~mL}$ of M9 minimal medium supplemented with $4 \mathrm{~g} / \mathrm{L}$ of glucose at $37^{\circ} \mathrm{C}$. Aliquots of growing $S$.

98 aureus were washed with phosphate-buffered saline three times, pelleted, and stored at $-80{ }^{\circ} \mathrm{C}$.

99 Boiling water $(500 \mu \mathrm{L})$ was added directly to the frozen pellets, and the samples were boiled for

10015 min to eliminate proteolytic activity following the methods of a previous study (Slavoff et al.,

101 2013). After cooling to room temperature, the samples were sonicated on ice for $20 \mathrm{~s}$. Acetic

102 acid was then added to the lysate to a final concentration of $0.25 \%$ by volume and centrifuged at

$10320,000 \mathrm{~g}$ for $20 \mathrm{~min}$ at $4{ }^{\circ} \mathrm{C}$. The supernatant was filtered by a $30-\mathrm{kDa}$ molecular weight cut-off

104 filter (Millipore). The filtered endogenous peptides were quantified by the BCA assay, desalted

105 using StageTip (Thermo Fisher Scientific), and used for mass spectrometry analysis.

106 LC-MS analysis 
107 Two $\mu \mathrm{g}$ of endogenous peptides of S. aureus were analyzed using an LTQ Orbitrap Velos

108 (Thermo Finnigan, San Jose, CA). First, the peptides were loaded into the precolumn $(75 \mu \mathrm{m} \times 2$

$109 \mathrm{~cm}$, Acclaim Pep Map 100 C18 column, $3 \mu \mathrm{m}, 100$ A; Thermo Fisher Scientific) at a flow rate of

$11010 \mu \mathrm{L} / \mathrm{min}$ and then were transferred to an analytical column $(75 \mu \mathrm{m} \times 25 \mathrm{~cm}$, Acclaim Pep Map

111 RSLC C18 column, $2 \mathrm{~m}, 100 \mathrm{~A}$; Thermo Fisher Scientific) at a flow rate of $300 \mathrm{~nL} / \mathrm{min}$. The

112 peptides were reverse-phase separated with buffer A ( $0.51 \%$ acetic acid) and buffer B (100\%

113 ACN and $0.1 \%$ acetic acid) under a 95 -min gradient (3-5\% buffer B for $5 \mathrm{~s}, 5-15 \%$ buffer B for

$11440 \mathrm{~min}, 15-28 \%$ buffer B for $34 \mathrm{~min}$ and $50 \mathrm{~s}, 28-38 \%$ buffer B for $12 \mathrm{~min}, 38-100 \%$ buffer B

115 for $5 \mathrm{~s}$, and $100 \%$ buffer B for $8 \mathrm{~min}$ ). MS survey scans were performed for mass-to-charge

116 ratios ranging from $350-1,800$, and the 20 most intense ions from the survey scans were analyzed

117 by MS/MS spectra in the LTQ, which were determined using Xcalibur mass spectrometer

118 software in real-time. With siloxane ( $\mathrm{m} / \mathrm{z} 445.120025)$ as a lock mass, dynamic mass exclusion

119 windows of $60 \mathrm{~s}$ were used similar to previous studies (Wang et al., 2015; Zhou et al., 2015).

120 Processing of MS data

121 Raw files were processed using MaxQuant (version 1.2.2.5) (Cox and Mann, 2008) and searched

122 against the UniProtStaphylococcus NCTC8325 protein database (2015/12/13; UniProtKB:

123 UP000008816; 2,889 sequences). Enzyme specificity was set to unspecific. The minimum

124 peptide length required was six amino acids. Variable modifications included oxidation (Met)

125 and acetylation (protein $\mathrm{N}$-terminus). Mass tolerances were set at $20 \mathrm{ppm}$ for precursor ions and 
$1260.5 \mathrm{Da}$ for fragment ions. An estimate of the false discovery rate (FDR) of the identification was

127 obtained using the reversed sequences in the databases. All FDRs were set to 0.01 for site,

128 peptide, and protein.

\section{Bioinformatics analysis of the endogenous peptidome}

130 To annotate the features of the peptidome, EMBOSS (Rice et al., 2000) was used to calculate the

131 isoelectric point $(\mathrm{pI})$ and molecular weight $(\mathrm{Mw})$ of the peptide sequences. One-way ANOVA

132 was performed to analyze the differential expression of peptides among groups, and a peptide

133 was considered differentially expressed if the FDR-q value was less than 0.01 . To analyze the

134 sequence patterns of proteases to generate endogenous peptides, the amino acid sequences

135 surrounding the termini of peptides were extracted, and overrepresented amino acids were

136 analyzed using iceLogo (Colaert et al., 2009). Overrepresented sequence motifs were analyzed

137 using motif-x (Chou and Schwartz, 2011; Schwartz and Gygi, 2005), and a P value of less than

1380.0001 was considered significant. Interactions among precursor proteins were annotated by the

139 STRING database (Szklarczyk et al., 2017), and the protein-protein interaction network was

140 constructed using Cytoscape software (Kohl et al., 2011). The database for annotation,

141 visualization, and integrated discovery (DAVID) (version 6.8) (Huang da et al., 2009) was used

142 to identify significantly enriched gene ontology terms in biological processes (BP), molecular

143 function (MF), and cellular component (CC) categories for the precursor proteins of the DE

144 endogenous peptidome. 
145 Results

146 Identification of endogenous peptides from $S$. aureus

147 To characterize the endogenous peptides of MSSA and MRSA, three clones from MSSA (ATCC

14829213 strains), three clones from MRSA (ATCC 43300 strains), and three S. aureus clinical

149 MRSA isolates were boiled before protein extraction to ensure that proteases were inactivated

150 and that all peptides identified were $S$. aureus endogenous peptides (i.e. not artificial proteolytic

151 peptides produced during or after protein extraction). To identify small endogenous peptides, the

152 proteins were filtered using a membrane with a molecular weight cut-off of $30 \mathrm{kD}$ so that only

153 small endogenous peptides remained. The extracted endogenous peptides were separated by

154 liquid chromatography and identified by an LTQ Orbitrap mass spectrometer. We selected high-

155 score reliable sequences with an FDR of $1 \%$ for peptides to ensure the reliability of the

156 proteomic identification. Filtering the reverse sequences ( 2 peptides) and contaminant proteins

157 (23 peptides) resulted in 1040 endogenous peptides, corresponding to 282 precursor proteins

158 from $S$. aureus. The detailed list of identified endogenous peptides is shown in Table S1. The $S$.

159 aureus has complex endogenous peptides.

160 Properties of the endogenous peptidome of $S$. aureus

161 With the identified endogenous peptidome, we then analyzed the features of these endogenous

162 peptide sequences. We found that some peptides are shorter forms of longer peptides. For 
163 example, there were 6 peptides for precursor protein P48940, including

164 "ANEILDAANNTGGAVK", "NEILDAANNTGG", "NEILDAANNTGGAVK",

165 "EILDAANNTGGAVK", "LDAANNTGGAVK" and "ILDAANNTGGAVK". The latter five

166 peptides occurred after the first and longest one. The longest peptide

167 “ANEILDAANNTGGAVK" was preserved for the bioinformatics analysis to facilitate

168 subsequent analyses. After filtering, the 274 representative (longest) peptides were obtained.

169 With the filtered peptides, we analyzed the length distribution and found that the peptides ranged

170 from 8 AA to 25 AA (Fig 1A), with 160 peptides between 10 AA and 13 AA. The length of the

171 endogenous peptides peaked at 11 AA. The endogenous peptides in $S$. aureus are relatively short

172 in length.

173 We further analyzed the isoelectric point and molecular weights of the endogenous peptides. The

174 isoelectric point distribution analysis of the endogenous peptidome of $S$. aureus revealed a wide

175 range of isoelectric point values from 3.05 to 11.82 (Fig 1B). The median of the isoelectric point

176 was 4.50 , indicating that many of the peptides were acidic. The molecular weights of the

177 identified peptides ranged from 759.41 to 2862.28 Da with a median of $1229.13 \mathrm{Da}$ (Fig 1C). A

178 two-dimensional plot of isoelectric point and molecular weight showed that most of the

179 endogenous peptides had molecular weights less than $2 \mathrm{kDa}$. (Fig 1D). These results suggest that

180 the endogenous peptides are mostly short, and are mainly acidic.

181 Differential expression of endogenous peptides among $S$. aureus strains 
182 We further analyzed differences of endogenous peptides among the different strains of S. aureus.

183 Statistical analysis showed that there were 435 DE endogenous peptides between MSSA and

184 MRSA (Table S2). Unsupervised hierarchical cluster analysis of the DE endogenous peptides

185 revealed 6 categories (Fig 2). Cluster C1 (140 peptides) and cluster C2 (152 peptides) were

186 highly expressed in MSSA. Peptides in cluster C3 (27 peptides), cluster C4 (58 peptides), and

187 cluster C5 (23 peptides) were highly expressed in both MSSA and MRSA with differential

188 expression patterns between standard MRSA and clinical isolates. Peptides in cluster C6 (35

189 peptides) were highly expressed in MRSA. Endogenous peptides from C1 and C2 (292 peptides)

190 highly expressed in MSSA were designated as MSSA-abundant endogenous peptides (MSSA-

191 AEP), and 35 peptides from C6 were designated as MRSA-abundant endogenous peptides

192 (MRSA-AEP). Differences in the expression of endogenous peptides between MRSA and MSSA

193 may be related to their physiological and functional differences.

194 Proteolytic cleavage features of MSSA-AEP and MRSA-AEP

195 DE peptides may be generated by differential protease activities between MSSA and MRSA. To

196 investigate the differential protease activities, precursor protein cleavage sites of peptides were

197 examined for the frequency of signature amino acids. We extracted sequences of 6 amino acids

198 surrounding the precursor proteins of MSSA-AEP and MRSA-AEP and analyzed the amino acid

199 distribution at the N-terminus and C-terminus of the DE peptides. Fig 3A shows an enrichment

200 diagram of amino acids around the N-terminus of MSSA-AEP. We found that "A", "S" and "D" 
201 were significantly enriched at the first amino acid of the peptides at the N-terminus; "A" and "S"

202 were significantly enriched at the P1 position; and "A", "T", "G” and "Q" were significantly

203 enriched at the $\mathrm{P} 1$ ' position $(\mathrm{p}<0.05)$. "Q", "A" and "N" were the most frequently observed

204 amino acids at the C- terminal end of the peptides. "Q" was significantly enriched at the P1' and

205 P1 positions; "A", "S" and "Q" were significantly enriched at the P1 position; and "Q", "L", "V"

206 and "Y" were significantly enriched at the $\mathrm{P} 1$ ' position $(\mathrm{p}<0.05)$ (Fig 3B). Fig 3C and 3D show

207 the enrichment diagram of amino acids around the N-terminus and C-terminus of MRSA-AEP.

208 We found that "I" was enriched at the first amino acid of the peptides at the N-terminus; there

209 was no enrichment of amino acids at the P1 position; and " $\mathrm{C}$ " was significantly enriched at the

210 P1' position $(\mathrm{p}<0.05)$. "A", "Q" and "V" were the most frequently observed amino acids at the

211 C-terminal end of the peptides; " $\mathrm{A}$ " and "Q" were significantly enriched at the start of the

212 peptides, "A" and "M" were significantly enriched at the P1 position, and "Q" was significantly

213 enriched at the $\mathrm{P} 1$ ' position $(\mathrm{p}<0.05)$. The uneven distribution of amino acids surrounding the

214 DE endogenous peptides indicated possible protease recognition motifs.

215 Given that the above sequence logo analysis may be subject to background amino acid

216 distribution, we used motif-x to examine the enrichment of amino acid residues. In-depth motif

217 analysis could help identify how proteolysis generates these peptides. We identified the N-

218 terminus and C-terminus of MSSA-AEP and MRSA-AEP and found that only the C-terminus of

219 the MSSA-AEP had an enriched motif ("VXXXK") (Fig 3E, Table S3). There was a valine at P0 
220 and lysine at P4. Seven peptides in MSSA contained this motif; among them, the precursor

221 protein corresponding to the sequence "TFTVDGVRFTKGQ" was Q2FVV8 (a putative

222 transcriptional regulator), and the corresponding precursor protein of the sequence

223 “DSNYTSVKDLKDN" was Q2FVX4 (molybdenum ABC transporter, periplasmic molybdate-

224 binding protein). No enrichment of the proteolytic motif was observed at the end of MRSA-AEP.

225 Therefore, these DE peptides may be produced by differential protease activities between MSSA 226 and MRSA.

\section{Annotations of the precursor proteins of MSSA-AEP and MRSA-AEP}

228 To better understand the potential functions of DE endogenous peptides, we used STRING to

229 analyze protein-protein interactions between precursor proteins of MSSA-AEP and MRSA-AEP,

230 and the precursors showed complex interactions (Fig 4). The peptides from ribosomal-related

231 precursor proteins, which show strong interactions, were highly expressed. These peptides might

232 regulate the functions of precursor proteins and participate in the regulation of translation

233 activities.

234 To further annotate their functions, the corresponding precursor proteins of MSSA-AEP and

235 MRSA-AEP were subjected to gene ontology and KEGG analyses (Table S4). Gene ontology

236 annotation revealed significant enrichment of precursor proteins in "translation" (14 peptides),

237 "structural constituent of ribosome" (13 peptides), and "rRNA binding" (9 peptides), indicating 
238 that many precursor proteins were involved in ribosome biogenesis or translational regulation

239 (Fig 5A), which is consistent with the results shown in the protein interaction network diagram

240 of the precursor proteins. rpsR, rpsC, rpsD, rpsH, rplA, rplJ, rplQ, rplR, rplU, rplX, rplC, rplD,

241 and gatB were the precursor proteins with ribosome function in MSSA-AEP, and rplQ and rpmI

242 were the precursor proteins in MRSA-AEP (Fig 4). The expression of peptides from rplQ was

243 much lower in MSSA-AEP than in MRSA-AEP; thus, we considered rpIQ to be the precursor

244 protein of MRSA-AEP. These ribosomal endogenous peptides may regulate the translational

245 efficiency (Cotter et al., 2005; Repka et al., 2017) and be responsible for the proteome

246 differences between MSSA and MRSA.

247 KEGG pathway analysis also revealed that these DE peptides were significantly enriched in 248 various metabolic pathways, such as carbon metabolism (8 peptides), citric acid cycle (TCA 249 cycle) (4 peptides) (Fig 5B). In addition, DE endogenous peptides were involved in $\beta$-lactam 250 resistance. All relevant precursor proteins were expressed by MSSA-AEP, and PBP1, PBP1a/2,

251 and PBP3 were penicillin-binding proteins (Fig 5C), which is consistent with "penicillin-

252 binding" in the gene ontology analysis. Thus, MSSA-AEP and MRSA-AEP are related to the 253 differences in metabolism, pathogenicity, and drug resistance between MSSA and MRSA.

\section{Discussion}


255 The regulation of proteins have been studied a lot in S. aureus (Becher et al., 2009; Zhao et al.,

256 2017). Small peptides (e.g., neuropeptides) are able to play important functions (Fridjonsdottir et

257 al., 2018), However, small peptides, especially the endogenous peptides, are less studied in $S$.

258 aureus. In this study, a proteomic analysis was conducted to characterize the endogenous

259 peptides of S. aureus (Schrader, 2018; Vincent, 2000) and identified 292 MSSA-AEP and 35

260 MRSA-AEP, indicating different endogenous peptidomes between MRSA and MSSA.

261 Endogenous peptides can be produced by proteolytic enzymes (Wang et al., 2012). We analyzed

262 the features of peptide sequences near the proteolytic N-terminal and C-terminal sites of MSSA-

263 AEP and MRSA-AEP and observed a non-random distribution of amino acids. Specifically, we

264 found that the amino acid distribution of MRSA-AEP and MRSA-AEP was uneven, and the

265 amino acid enrichment of MRSA-AEP was significantly reduced. The C-terminus of MSSA-

266 AEP was enriched in specific amino acid residues, potentially reflecting protease activity during

267 peptide maturation, while MRSA-AEP did not have an enriched motif because there were fewer

268 peptides identified in MRSA than MSSA. Deeper peptide coverage may help us discover motifs.

269 The motif analysis suggested that endogenous peptides were not randomly broken and were

270 regulated by proteases. However, the exact enzyme recognition motif is still unknown due to the

271 lack of research on the substrate features of proteolytic enzymes. Additional studies to

272 characterize such protease would help explain the differential endogenous peptidome between

273 MSSA and MRSA. 
274 MRSA is resistant to methicillin, a type of beta-lactam antibiotic that causes bacteria to stop

275 dividing and die (Peacock and Paterson, 2015). The KEGG analysis showed that the beta-lactam

276 resistance pathway was enriched in MSSA-AEP but not MRSA-AEP. In MSSA-AEP, peptides

277 from the penicillin-binding proteins PBP1, 1a/2, and 3, which have high affinities for beta-lactam

278 antibiotics (Fisher and Mobashery, 2021), may be involved in its sensitivity to penicillin.

279 In addition, analysis of the precursor proteins of MSSA-AEP and MRSA-AEP by gene ontology

280 analysis revealed the enrichment of ribosome or translational regulation, which is consistent with

281 the observed complex interactions among ribosomal proteins in the protein-protein interaction

282 network analysis. We found that there were 21 ribosomal peptides in MSSA-AEP, and only 4

283 ribosomal peptides in MRSA-AEP. It is widely accepted that ribosomes can regulate cell growth

284 and metabolism (Fu et al., 2013; Jiang et al., 2015). For example, 50S ribosomal protein L1

285 (rplA) is a ribosomal structural component highly expressed in MSSA-AEP. L1 has dual

286 functions as a ribosomal structural protein that binds rRNA and as a translational repressor that

287 binds its mRNA (Nevskaya et al., 2006) and is related to bacterial growth (Dean and Nomura,

288 1980). Many antibiotics interfere with protein synthesis and function by binding to ribosomes

289 and thereby inhibiting bacterial reproduction (Foster, 2017). In comparison with MRSA-AEP,

290 MSSA-AEP have stronger ribosome-related functions, and might be involved in the regulation of

291 bacterial growth. 
292 We also observed that the precursor proteins of MRSA-AEP were mainly enriched in

293 metabolism pathways, including the TCA cycle. The activity of the TCA cycle is related to the

294 virulence and survival of pathogens, the production of the substances of biofilm mucus,

295 intercellular adhesin, and persistent infection (Gao and Stewart, 2004; Oscarsson et al., 2006).

296 For example, one peptide among the MRSA-AEP is derived from the branched-chain alpha-keto

297 acid dehydrogenase subunit E2 (SAOUHSC_01042), which is essential in the adhesion of $S$.

298 aureus to host eukaryotic cells and their survival (Khairon et al., 2016). Aside from proteins in

299 the TCA cycle, we found that MRSA-AEP were also derived from other metabolic processes,

300 such as one peptide from fructose-1,6-diphosphate aldehydes (fda) that is closely related to the

301 viability of pathogenic microorganisms (Yadav et al., 2013). Two MRSA-AEP were derived

302 from CopZ, the excess of which causes bacterial toxicity and affects the growth, vitality, and

303 metabolism of bacteria (Stewart et al., 2020; Xu et al., 2021). The phenotypic changes of MRSA,

304 such as the formation of smaller colonies and the ability to colonize for a longer period, may be

305 related to its metabolic changes. These changes in metabolism pathways of MRSA-AEP are

306 associated with antibiotic resistance and increased toxicity.

307 By analyzing MSSA-AEP precursor proteins, we also found that that many of them were

308 involved in immunity regulation. For example, S. aureus recombinant alkaline shock protein 23

309 (Asp23) has immunogenicity and protective effects and is a promising vaccine candidate as a

310 prophylactic therapeutic agent for $S$. aureus (Francis and Kuyyalil, 2018). Immunodominant 
311 staphylococcal antigen A (isaA), which is expressed on the surface of the bacterial cell wall,

312 plays a role in immunogenicity in the cleavage of peptidoglycan and the inhibition of biofilm

313 formation (Valliammai et al., 2020). ESAT-6 secretion accessory factor (esaA) and ESAT-6

314 secretion machinery protein (essB) are immunogenic antigens, both of which can induce a strong

315 immune response and have protective effects against bacterial infection (Klein et al., 2021; Ma et

316 al., 2020). The peptide derived from ESAT-6, essB, and esaA may also be able to induce

317 immune responses when secreted.

\section{Conclusion}

319 In sum, our characterization of the DE endogenous peptides between MSSA and MRSA revealed

320 their complex regulatory roles in antibiotic resistance, bacterial survival, and immune responses.

321 Additional functional studies of these DE endogenous peptides between MSSA and MRSA are

322 needed to help elucidate the mechanisms underlying their pathogenicity, phenotypes, and

323 differences in antibiotic resistance.

\section{Data Availability Statement}

325 The mass spectrometry proteomics data have been deposited to the ProteomeXchange

326 Consortium via the MassIVE partner repository (MassIVE Dataset Summary (ucsd.edu)) with

327 the dataset identifier MSV000088118.

\section{Author Contributions}


329 Y.L. and D.S. contributed to the conceptualization of the model. Haixia Tu, Yan Li and Fei Xu

330 wrote the manuscript. Yiwei Cheng, QiangLong Pan, XiaoCai, Shuting Ge, Shouxing Wang and

331 Min Cao provided experimental materials and helped with the experimental analysis. All authors

332 read and approved the final manuscript.

\section{Conflict of Interest}

334 The authors declare that the research was conducted in the absence of any commercial or

335 financial relationships that could be construed as a potential conflict of interest.

\section{Funding}

337 This work was supported by grant from the Sir Run Run Hospital, Nanjing Medical University

338 (YFZDXK01-5) and the School Foundation of Nanjing Medical University (NMUB2020319).

\section{Supplementary Material}

340 Figure 1. Distribution of physicochemical features of the endogenous peptidome of S. aureus.

341 The count distribution of peptide length (A), isoelectric point (B), and molecular weight (C) are

342 given separately for all identified endogenous peptides of $S$. aureus. The two-dimensional

343 distribution of the isoelectric point and molecular weight of the identified peptides is shown in 344 (D). 
345 Figure 2. Cluster analysis of the DE endogenous peptidome between MSSA and MRSA.

346 Unsupervised hierarchical clustering analysis of all DE endogenous peptides from MSSA

347 (ATCC 29213), MRSA (ATCC 43300), and S. aureus clinical isolates (MRSA-p1 to -p3).

348 Figure 3. The sequence logos and enrichment of amino acids and motifs surrounding the termini

349 of MSSA-AEP and MRSA-AEP. The N-terminal consensus sequences (A) and C-terminal

350 consensus sequences (B) of MSSA-AEP, and the N-terminal consensus sequence (C) and C-

351 terminal consensus sequence (D) of MRSA-AEP. According to the motif-x analysis, there was

352 one motif $(\mathrm{E})$ that was enriched in the $\mathrm{C}$-terminal sequences of MSSA-AEP $(\mathrm{P}<0.0001)$.

353 Different types of amino acids highlighted in different colors correspond to their annotations.

354 Figure 4. Protein-protein interaction network annotations of DE endogenous peptides between

355 MSSA and MRSA strains by the STRING database. The protein-protein interactions were

356 annotated from the STRING database and visualized by Cytoscape. The red nodes represent the

357 translation-related proteins and ribosomal components in the precursor proteins of MRSA-AEP,

358 while the blue nodes represent the translation-related proteins and ribosomal components in the

359 precursor proteins of MSSA-AEP. The edges represent protein-protein associations from the

360 STRING database.

361 Figure 5. The gene ontology and KEGG enrichment analysis of precursor proteins of MSSA-

362 AEP and MRSA-AEP. (A) Enriched gene ontology terms for biological process (BP), cellular 
363

364 365 366 367 368

component $(\mathrm{CC})$, and molecular function $(\mathrm{MF})$ are shown together with the gene ratio. (B)

Enriched KEGG pathways. (C) Beta-lactam resistance pathway red stars represent pathwayrelated precursor proteins in MSSA-AEP.

Table S1. Information of the endogenous peptides identified by mass spectrometry in S. aureus.

Table S2. The properties of DE endogenous peptides between MSSA and MRSA.

Table S3. Sequences matching the enriched motif at the C-terminus in MSSA-AEP.

Table S4. Enriched gene ontology terms and KEGG pathways in DE endogenous peptides between MSSA and MRSA.

\section{References:}

Akil, N., Muhlebach, M.S. (2018) Biology and management of methicillin resistant Staphylococcus aureus in cystic fibrosis. Pediatr Pulmonol 53, S64-S74. Becher, D., Hempel, K., Sievers, S., Zuhlke, D., Pane-Farre, J., Otto, A., Fuchs, S., Albrecht, D., Bernhardt, J., Engelmann, S., Volker, U., van Dijl, J.M., Hecker, M. (2009) A proteomic view of an important human pathogen--towards the quantification of the entire Staphylococcus aureus proteome. PLoS One 4, e8176.

Carter, A.M. (2012) Evolution of placental function in mammals: the molecular basis of gas and nutrient transfer, hormone secretion, and immune responses. Physiol Rev 92, 1543-1576. Cheung, G.Y., Joo, H.S., Chatterjee, S.S., Otto, M. (2014) Phenol-soluble modulins--critical determinants of staphylococcal virulence. FEMS Microbiol Rev 38, 698-719.

Chou, M.F., Schwartz, D. (2011) Biological sequence motif discovery using motif-x. Curr Protoc Bioinformatics Chapter 13, Unit 13 15-24.

Colaert, N., Helsens, K., Martens, L., Vandekerckhove, J., Gevaert, K. (2009) Improved visualization of protein consensus sequences by iceLogo. Nat Methods 6, 786-787.

Cordwell, S.J., Larsen, M.R., Cole, R.T., Walsh, B.J. (2002) Comparative proteomics of Staphylococcus aureus and the response of methicillin-resistant and methicillin-sensitive strains to Triton X-100. Microbiology 148, 2765-2781. 
389 Cotter, P.D., Hill, C., Ross, R.P. (2005) Bacteriocins: developing innate immunity for food. Nat 390 Rev Microbiol 3, 777-788.

391 Cox, J., Mann, M. (2008) MaxQuant enables high peptide identification rates, individualized

392 p.p.b.-range mass accuracies and proteome-wide protein quantification. Nat Biotechnol 26, 393 1367-1372.

394 Cui, X., Li, Y., Yang, L., You, L., Wang, X., Shi, C., Ji, C., Guo, X. (2016) Peptidome analysis 395 of human milk from women delivering macrosomic fetuses reveals multiple means of protection 396 for infants. Oncotarget 7, 63514-63525.

397 Dean, D., Nomura, M. (1980) Feedback regulation of ribosomal protein gene expression in 398 Escherichia coli. Proc Natl Acad Sci U S A 77, 3590-3594.

399 Fisher, J.F., Mobashery, S. (2021) beta-Lactams against the Fortress of the Gram-Positive 400 Staphylococcus aureus Bacterium. Chem Rev 121, 3412-3463.

401 Foster, T.J. (2017) Antibiotic resistance in Staphylococcus aureus. Current status and future 402 prospects. FEMS Microbiol Rev 41, 430-449.

403 Francis, D., Kuyyalil, S. (2018) Immunogenicity and protective efficacy of recombinant alkaline 404 shock protein 23 from Staphylococcus aureus in a murine model. Cent Eur J Immunol 43, 371405377.

406 Fricker, L.D. (2018) Carboxypeptidase E and the Identification of Novel Neuropeptides as 407 Potential Therapeutic Targets. Adv Pharmacol 82, 85-102.

408 Fridjonsdottir, E., Nilsson, A., Wadensten, H., Andren, P.E. (2018) Brain Tissue Sample 409 Stabilization and Extraction Strategies for Neuropeptidomics. Methods Mol Biol 1719, 41-49.

410 Fu, Y., Deiorio-Haggar, K., Anthony, J., Meyer, M.M. (2013) Most RNAs regulating ribosomal 411 protein biosynthesis in Escherichia coli are narrowly distributed to Gammaproteobacteria.

412 Nucleic Acids Res 41, 3491-3503.

413 Gao, J., Stewart, G.C. (2004) Regulatory elements of the Staphylococcus aureus protein A (Spa) 414 promoter. J Bacteriol 186, 3738-3748.

415 Garcia, A.B., Vinuela-Prieto, J.M., Lopez-Gonzalez, L., Candel, F.J. (2017) Correlation between 416 resistance mechanisms in Staphylococcus aureus and cell wall and septum thickening. Infect 417 Drug Resist 10, 353-356.

418 Gjertsson, I., Jonsson, I.M., Peschel, A., Tarkowski, A., Lindholm, C. (2012) Formylated 419 peptides are important virulence factors in Staphylococcus aureus arthritis in mice. J Infect Dis 420 205, 305-311.

421 Huang da, W., Sherman, B.T., Lempicki, R.A. (2009) Systematic and integrative analysis of 422 large gene lists using DAVID bioinformatics resources. Nat Protoc 4, 44-57.

423 Jeong, H., Kang, S., Cho, H.J. (2020) Prevalence of Multidrug-Resistant Organisms and Risk 424 Factors for Carriage among Patients Transferred from Long-Term Care Facilities. Infect 425 Chemother 52, 183-193.

426 Ji, X., Liu, X., Peng, Y., Zhan, R., Xu, H., Ge, X. (2017) Comparative analysis of methicillin427 sensitive and resistant Staphylococcus aureus exposed to emodin based on proteomic profiling. 
428 Biochem Biophys Res Commun 494, 318-324.

429 Jiang, N., Hu, L., Liu, C., Gao, X., Zheng, S. (2015) 60S ribosomal protein L35 regulates beta-

430 casein translational elongation and secretion in bovine mammary epithelial cells. Arch Biochem

431 Biophys 583, 130-139.

432 Kanlaya, R., Thongboonkerd, V. (2018) Quantitative peptidomics of endogenous peptides

433 involved in TGF-betal-induced epithelial mesenchymal transition of renal epithelial cells. Cell

434 Death Discov 4, 9.

435 Khairon, R., Zin, N.M., Abdul Rahman, M., Basri, D.F. (2016) Comparative Proteomic Analysis

436 of Differential Proteins in Response to Aqueous Extract of Quercus infectoria Gall in

437 Methicillin-Resistant Staphylococcus aureus. Int J Proteomics 2016, 4029172.

438 Klein, T.A., Grebenc, D.W., Gandhi, S.Y., Shah, V.S., Kim, Y., Whitney, J.C. (2021) Structure

439 of the Extracellular Region of the Bacterial Type VIIb Secretion System Subunit EsaA. Structure 440 29, 177-185 e176.

441 Kohl, M., Wiese, S., Warscheid, B. (2011) Cytoscape: software for visualization and analysis of 442 biological networks. Methods Mol Biol 696, 291-303.

443 Ma, Y., Hao, L., Liang, Z., Ma, J., Ke, H., Kang, H., Yang, H., Wu, J., Feng, G., Liu, Z. (2020)

444 Characterization of novel antigenic vaccine candidates for nile tilapia (Oreochromis niloticus)

445 against Streptococcus agalactiae infection. Fish Shellfish Immunol 105, 405-414.

446 Monteiro, R., Vitorino, R., Domingues, P., Radhouani, H., Carvalho, C., Poeta, P., Torres, C.,

447 Igrejas, G. (2012) Proteome of a methicillin-resistant Staphylococcus aureus clinical strain of

448 sequence type ST398. J Proteomics 75, 2892-2915.

449 Nevskaya, N., Tishchenko, S., Volchkov, S., Kljashtorny, V., Nikonova, E., Nikonov, O.,

450 Nikulin, A., Kohrer, C., Piendl, W., Zimmermann, R., Stockley, P., Garber, M., Nikonov, S.

451 (2006) New insights into the interaction of ribosomal protein L1 with RNA. J Mol Biol 355, 747-

452759.

453 Oscarsson, J., Tegmark-Wisell, K., Arvidson, S. (2006) Coordinated and differential control of

454 aureolysin (aur) and serine protease (sspA) transcription in Staphylococcus aureus by sarA, rot

455 and agr (RNAIII). Int J Med Microbiol 296, 365-380.

456 Otto, A., van Dijl, J.M., Hecker, M., Becher, D. (2014) The Staphylococcus aureus proteome. Int

457 J Med Microbiol 304, 110-120.

458 Otto, M. (2001) Staphylococcus aureus and Staphylococcus epidermidis peptide pheromones

459 produced by the accessory gene regulator agr system. Peptides 22, 1603-1608.

460 Peacock, S.J., Paterson, G.K. (2015) Mechanisms of Methicillin Resistance in Staphylococcus 461 aureus. Annu Rev Biochem 84, 577-601.

462 Repka, L.M., Chekan, J.R., Nair, S.K., van der Donk, W.A. (2017) Mechanistic Understanding 463 of Lanthipeptide Biosynthetic Enzymes. Chem Rev 117, 5457-5520.

464 Rice, P., Longden, I., Bleasby, A. (2000) EMBOSS: the European Molecular Biology Open

465 Software Suite. Trends Genet 16, 276-277.

466 Romanova, E.V., Sweedler, J.V. (2015) Peptidomics for the discovery and characterization of 
467 neuropeptides and hormones. Trends Pharmacol Sci 36, 579-586.

468 Schrader, M. (2018) Origins, Technological Development, and Applications of Peptidomics.

469 Methods Mol Biol 1719, 3-39.

470 Schwartz, D., Gygi, S.P. (2005) An iterative statistical approach to the identification of protein

471 phosphorylation motifs from large-scale data sets. Nat Biotechnol 23, 1391-1398.

472 Singh, J., Mumtaz, S., Joshi, S., Mukhopadhyay, K. (2020) In Vitro and Ex Vivo Efficacy of

473 Novel Trp-Arg Rich Analogue of alpha-MSH against Staphylococcus aureus. ACS Omega 5,

474 3258-3270.

475 Slavoff, S.A., Mitchell, A.J., Schwaid, A.G., Cabili, M.N., Ma, J., Levin, J.Z., Karger, A.D.,

476 Budnik, B.A., Rinn, J.L., Saghatelian, A. (2013) Peptidomic discovery of short open reading

477 frame-encoded peptides in human cells. Nat Chem Biol 9, 59-64.

478 Stewart, L.J., Ong, C.Y., Zhang, M.M., Brouwer, S., McIntyre, L., Davies, M.R., Walker, M.J.,

479 McEwan, A.G., Waldron, K.J., Djoko, K.Y. (2020) Role of Glutathione in Buffering Excess

480 Intracellular Copper in Streptococcus pyogenes. mBio 11.

481 Szklarczyk, D., Morris, J.H., Cook, H., Kuhn, M., Wyder, S., Simonovic, M., Santos, A.,

482 Doncheva, N.T., Roth, A., Bork, P., Jensen, L.J., von Mering, C. (2017) The STRING database

483 in 2017: quality-controlled protein-protein association networks, made broadly accessible.

484 Nucleic Acids Res 45, D362-D368.

485 Valliammai, A., Sethupathy, S., Ananthi, S., Priya, A., Selvaraj, A., Nivetha, V., Aravindraja, C.,

486 Mahalingam, S., Pandian, S.K. (2020) Proteomic profiling unveils citral modulating expression

487 of IsaA, CodY and SaeS to inhibit biofilm and virulence in methicillin-resistant Staphylococcus

488 aureus. Int J Biol Macromol.

489 Vincent, J.B. (2000) Elucidating a biological role for chromium at a molecular level. Acc Chem

490 Res 33, 503-510.

491 Wang, F., Zhu, J., Hu, L., Qin, H., Ye, M., Zou, H. (2012) Comprehensive analysis of the N and

$492 \mathrm{C}$ terminus of endogenous serum peptides reveals a highly conserved cleavage site pattern

493 derived from proteolytic enzymes. Protein Cell 3, 669-674.

494 Wang, J., Qi, L., Huang, S., Zhou, T., Guo, Y., Wang, G., Guo, X., Zhou, Z., Sha, J. (2015)

495 Quantitative phosphoproteomics analysis reveals a key role of insulin growth factor 1 receptor

496 (IGF1R) tyrosine kinase in human sperm capacitation. Mol Cell Proteomics 14, 1104-1112.

497 Wolf, E., Milazzo, S., Boehm, K., Zwahlen, M., Horneber, M. (2011) Thymic peptides for

498 treatment of cancer patients. Cochrane Database Syst Rev, CD003993.

499 Xu, F., Ke, X., Hong, M., Huang, M., Chen, C., Tian, X., Hang, H., Chu, J. (2021) Exploring the

500 metabolic fate of propanol in industrial erythromycin-producing strain via (13)C labeling

501 experiments and enhancement of erythromycin production by rational metabolic engineering of

502 Saccharopolyspora erythraea. Biochem Biophys Res Commun 542, 73-79.

503 Yadav, P.K., Singh, G., Gautam, B., Singh, S., Yadav, M., Srivastav, U., Singh, B. (2013)

504 Molecular modeling, dynamics studies and virtual screening of Fructose 1, 6 biphosphate

505 aldolase-II in community acquired- methicillin resistant Staphylococcus aureus (CA-MRSA). 
506 Bioinformation 9, 158-164.

507 Zhang, Y., Wang, Y., Van Driel, M.L., McGuire, T.M., Zhang, T., Dong, Y., Liu, Y., Liu, L.,

508 Hao, R., Cao, L., Xing, J., Dong, Y. (2019) Network meta-analysis and pharmacoeconomic

509 evaluation of antibiotics for the treatment of patients infected with complicated skin and soft

510 structure infection and hospital-acquired or ventilator-associated penumonia. Antimicrob Resist

511 Infect Control 8, 72.

512 Zhao, H., Hu, F., Yang, H., Ding, B., Xu, X., He, C., Cui, Z., Shu, W., Liu, Q. (2017) Isobaric

513 tags for relative and absolute quantitation proteomics analysis of gene regulation by SprC in

514 Staphylococcus aureus. Future Microbiol 12, 1181-1199.

515 Zhou, Q., Guo, Y., Zheng, B., Shao, B., Jiang, M., Wang, G., Zhou, T., Wang, L., Zhou, Z., Guo,

516 X., Huang, X. (2015) Establishment of a proteome profile and identification of molecular

517 markers for mouse spermatogonial stem cells. J Cell Mol Med 19, 521-534. 
Figure 1

Distribution of physicochemical features of the endogenous peptidome of $S$. aureus.

The count distribution of peptide length (A), isoelectric point (B), and molecular weight (C) are given separately for all identified endogenous peptides of $S$. aureus. The two-dimensional distribution of the isoelectric point and molecular weight of the identified peptides is shown in (D).

A

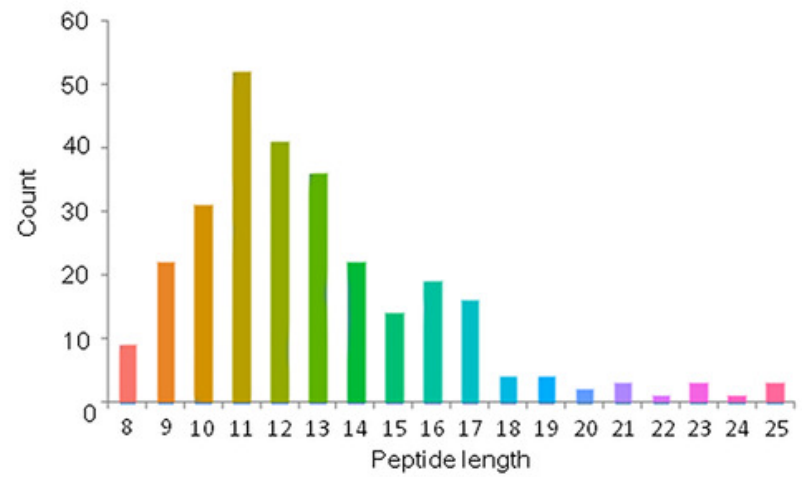

C

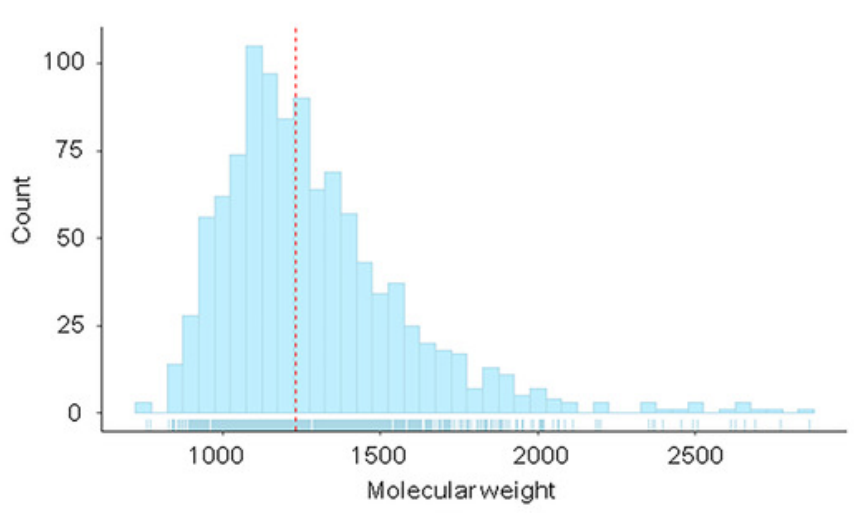

B

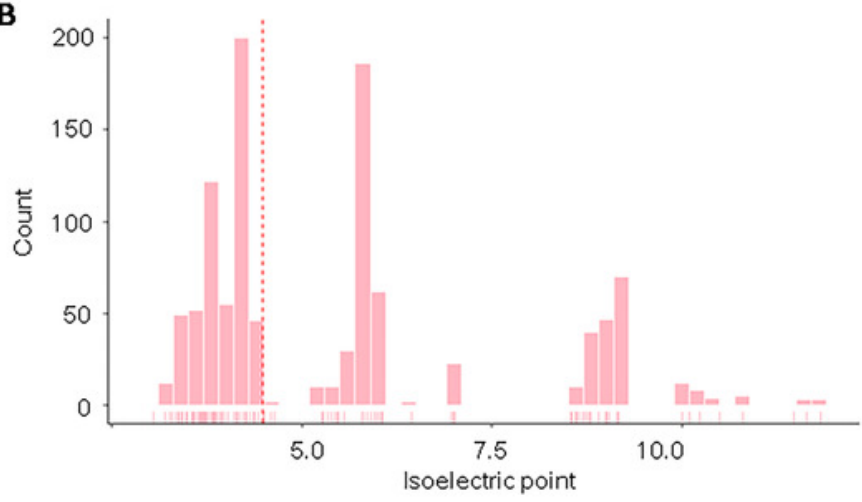

D

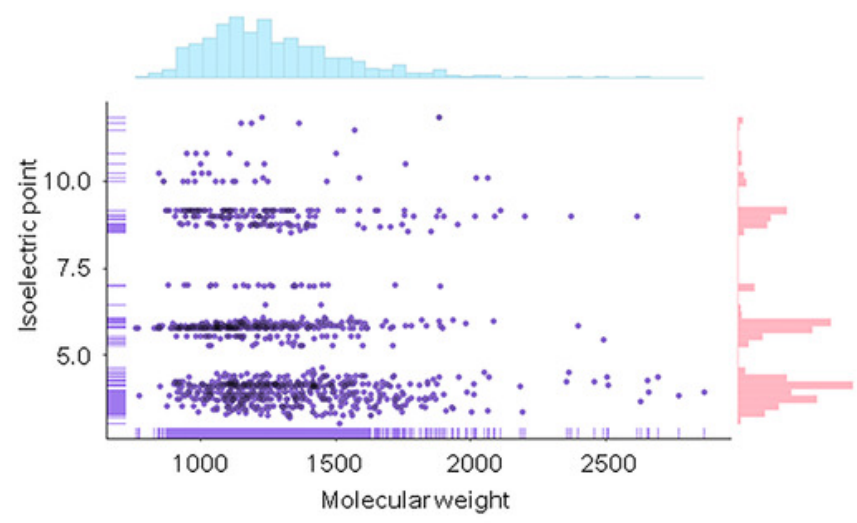


Figure 2

Cluster analysis of the DE endogenous peptidome between MSSA and MRSA.

Unsupervised hierarchical clustering analysis of all DE endogenous peptides from MSSA (ATCC 29213), MRSA (ATCC 43300), and S. aureus clinical isolates (MRSA-p1 to -p3).

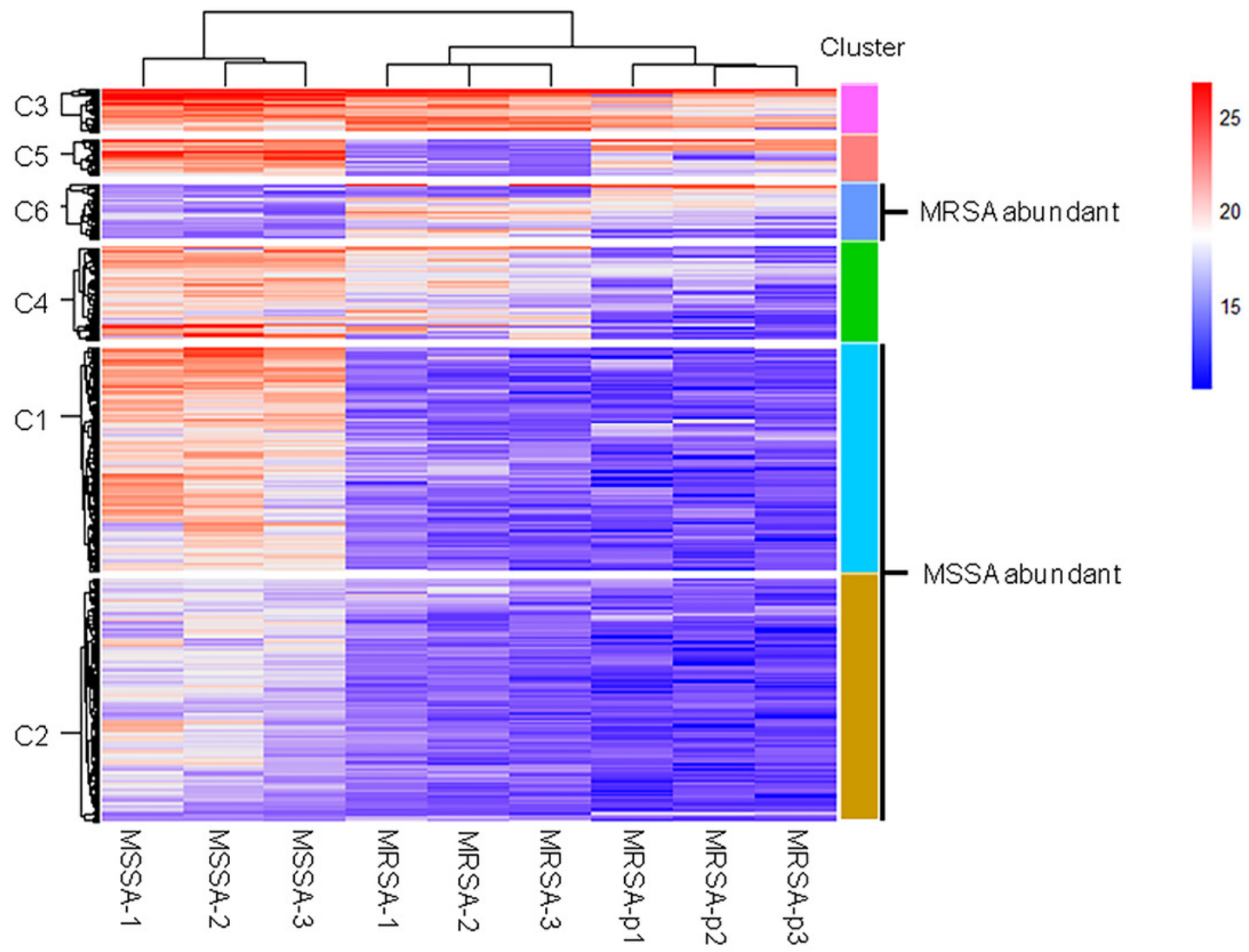


Figure 3

The sequence logos and enrichment of amino acids and motifs surrounding the termini of MSSA-AEP and MRSA-AEP.

The N-terminal consensus sequences (A) and C-terminal consensus sequences (B) of MSSA$A E P$, and the N-terminal consensus sequence $(C)$ and $C$-terminal consensus sequence (D) of MRSA-AEP. According to the motif- $x$ analysis, there was one motif (E) that was enriched in the $C$-terminal sequences of MSSA-AEP $(P<0.0001)$. Different types of amino acids highlighted in different colors correspond to their annotations.
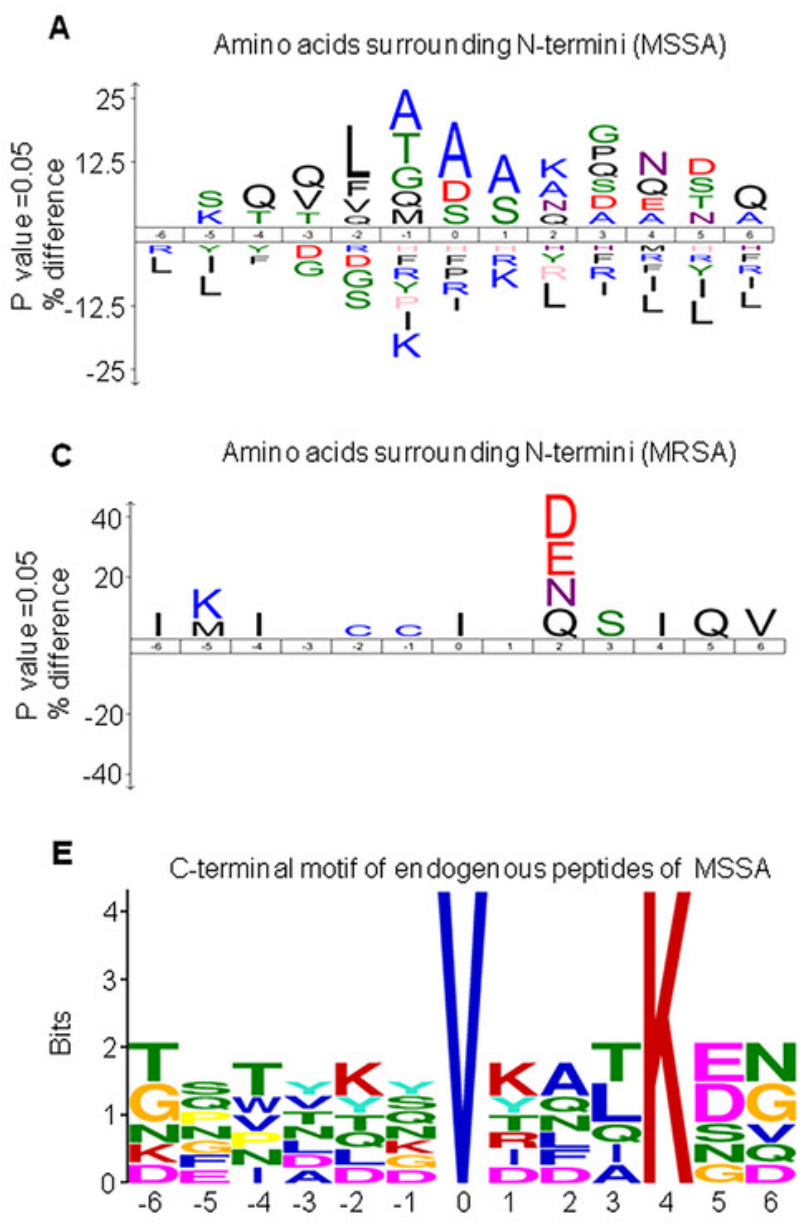

B Amino acids surrounding C-termini (MSSA)

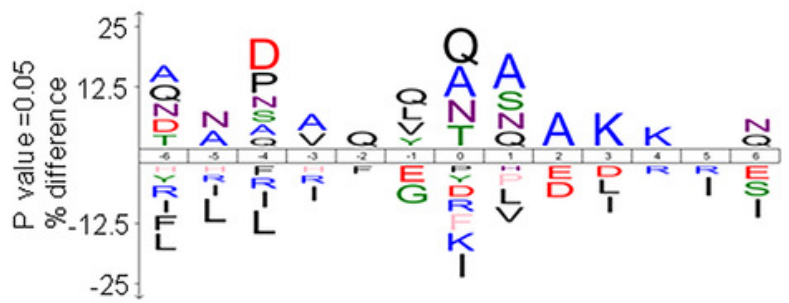

D Amino acids surrounding C-termini (MRSA)

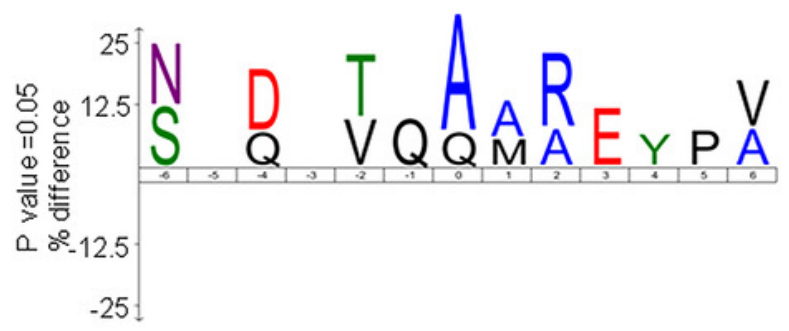




\section{Figure 4}

Protein-protein interaction network annotations of DE endogenous peptides between MSSA and MRSA strains by the STRING database.

The protein-protein interactions were annotated from the STRING database and visualized by Cytoscape. The red nodes represent the translation-related proteins and ribosomal components in the precursor proteins of MRSA-AEP, while the blue nodes represent the translation-related proteins and ribosomal components in the precursor proteins of MSSAAEP. The edges represent protein-protein associations from the STRING database.

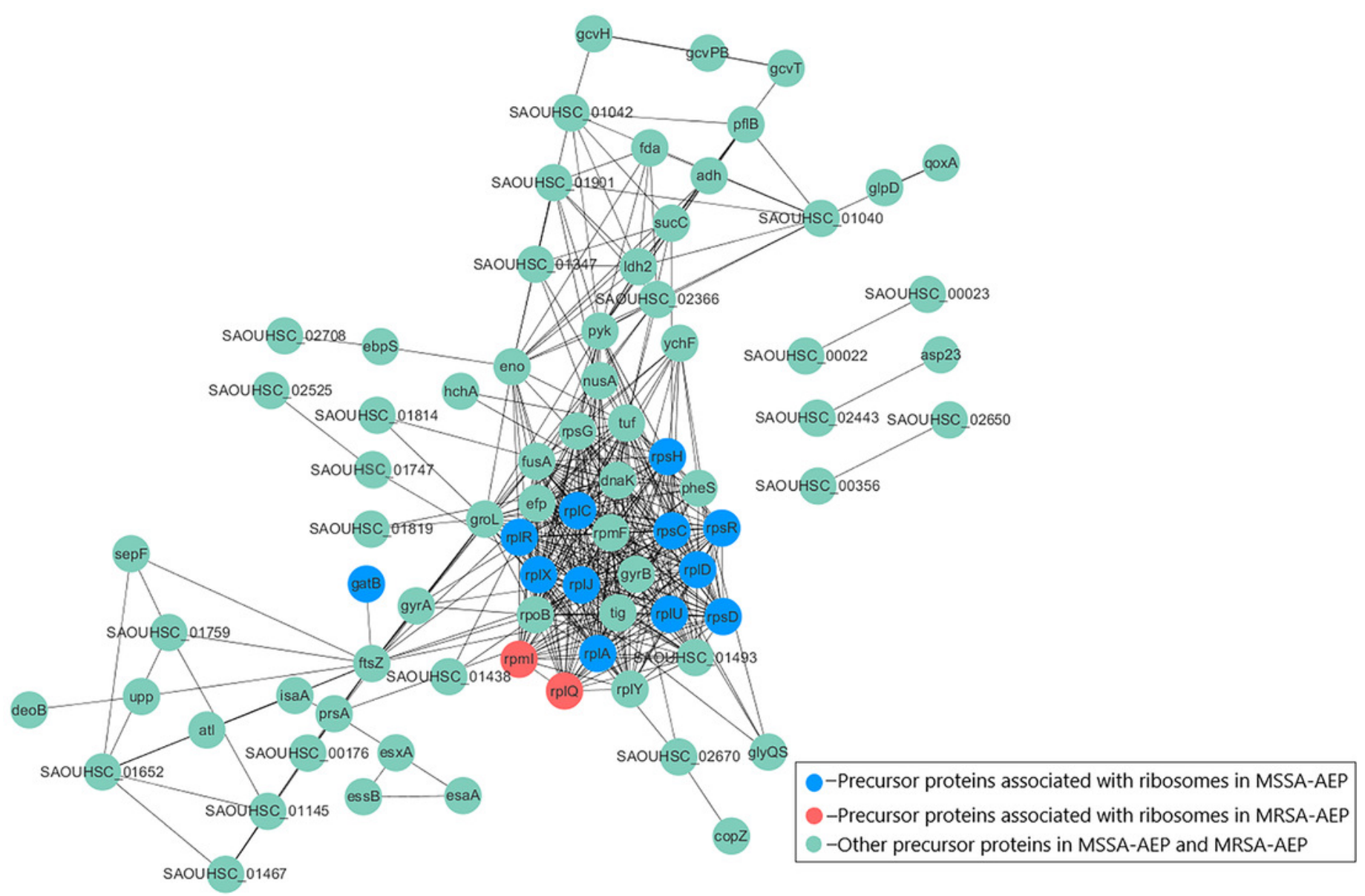




\section{Figure 5}

The gene ontology and KEGG enrichment analysis of precursor proteins of MSSA-AEP and MRSA-AEP.

(A) Enriched gene ontology terms for biological process (BP), cellular component (CC), and molecular function (MF) are shown together with the gene ratio. (B) Enriched KEGG pathways. (C) Beta-lactam resistance pathway red stars represent pathway-related precursor proteins in MSSA-AEP. 
A

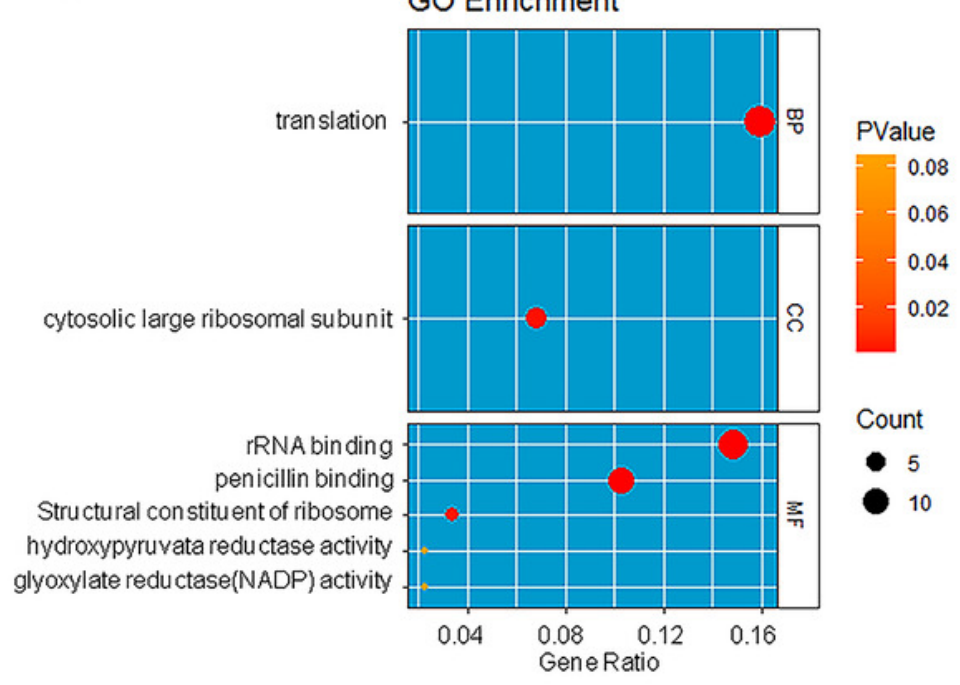

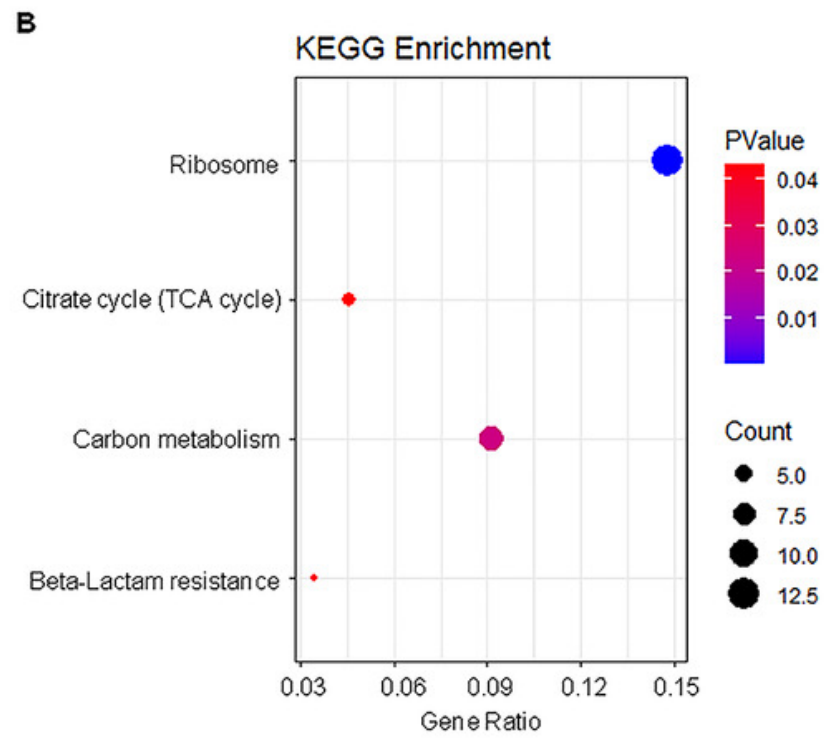

B

C

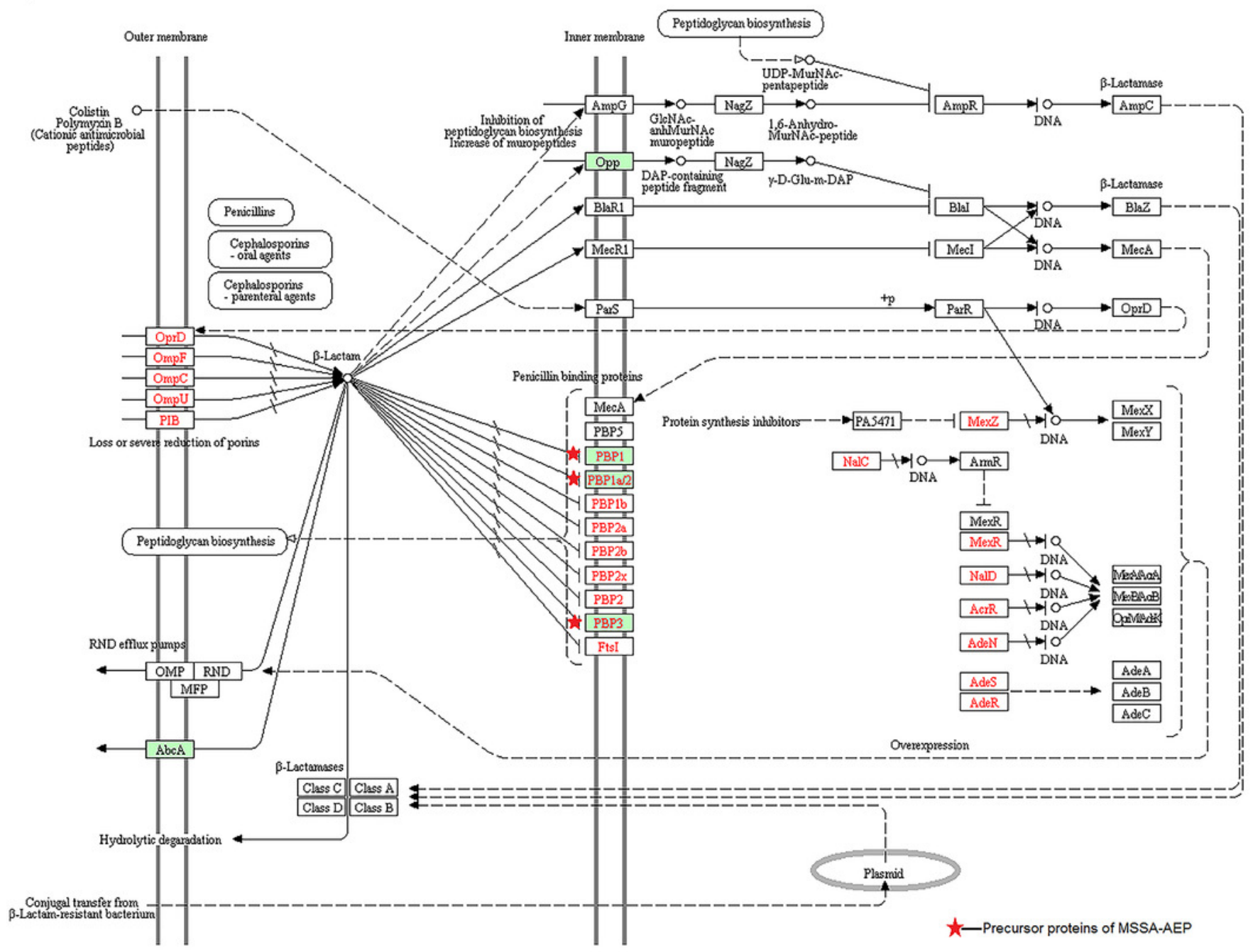

